\title{
Comparison of Ligand Architecture on Vapor Deposition Precursors: Synthesis, Characterization, and Reactivity of Volatile Cadmium Bis-Amidinate Complexes
}

\author{
Michael J. Foody, ${ }^{\dagger}$ Matthew S. Weimer, ${ }^{\dagger \neq}$ Harish Bhandari, ${ }^{\#}$ and Adam S. Hock ${ }^{\dagger *}$ \\ ${ }^{\dagger}$ Department of Chemistry, Illinois Institute of Technology, 3101 South Dearborn Street, \\ Chicago, Illinois 60616, United States. \\ \#Radiation Monitoring Devices, Inc., 44 Hunt Street, Watertown, MA 02472 \\ *Lam Research Corporation, 11155 SW Leveton Drive, Tualatin, Oregon 97062-8094, \\ United States
}

\section{Supplemental Materials}

Synthesis of Cd(amd)(SSiPh $)$ (4). $1(615.1 \mathrm{mg}, 1.6$ mmole) was dissolved in $10 \mathrm{ml}$ of toluene followed by the addition of triphenylsilanethiol $(430.0 \mathrm{mg}, 1.6 \mathrm{mmole})$ at room temperature. The reaction was allowed to stir at room temperature for 30 minutes. Volatile components were removed under reduced pressure to yield $840.1 \mathrm{mg}(99 \%)$ of a white solid. X-ray quality crystals could not be grown in toluene or diethyl ether. Mp: $88{ }^{\circ} \mathrm{C}$. Observed: ${ }^{1} \mathrm{H}$ NMR $\left(\mathrm{C}_{6} \mathrm{D}_{6}, 25{ }^{\circ} \mathrm{C}, \mathrm{ppm}\right): 8.04$ (br, 6H), 7.20 (br, 9H), 3.36 (br, 2H), 1.45 $(\mathrm{s}, 6 \mathrm{H}), .97(\mathrm{~d}, 12 \mathrm{H}) .{ }^{13} \mathrm{C} \mathrm{NMR}\left(\mathrm{C}_{6} \mathrm{D}_{6}, 25^{\circ} \mathrm{C}, \mathrm{ppm}\right): 154.0\left(\mathrm{C}_{\mathrm{q}}\right.$ - amidinate $), 136.01(\mathrm{CH}-$ phenyl), 127.72 ( $\mathrm{CH}$ - phenyl), $127.48\left(\mathrm{CH}\right.$ - phenyl), $46.1\left(\mathrm{CH}\right.$ - isopropyl), $25.05\left(\mathrm{CH}_{3}\right.$ - amidinate), $16.8\left(\mathrm{CH}_{3}\right.$ - isopropyl).

Both ${ }^{1} \mathrm{H}$ and ${ }^{13} \mathrm{C}$ NMR analyses showed solvent peaks corresponding to toluene even after pumping under vacuum for $8+$ hours at $50{ }^{\circ} \mathrm{C}$. Due to the excess solvent, clear elemental analysis was unable to confirm $\mathrm{CHN}$ analysis of pure 4. Purification through vacuum sublimation of $\mathbf{4}$ was unsuccessful. During sublimation, white crystalline solid began to accumulate on the sublimation cold finger when 4 was heated to $150{ }^{\circ} \mathrm{C}$ at 50 mTorr. However, when the sublimed product was analyzed, it was found to be pure $\mathbf{1}$. This suggests $\mathbf{4}$ disproportionates at elevated temperatures into $\mathbf{1}$ and an uncharacterized cadmium thiolate compound, which was not soluble in organic solvent.

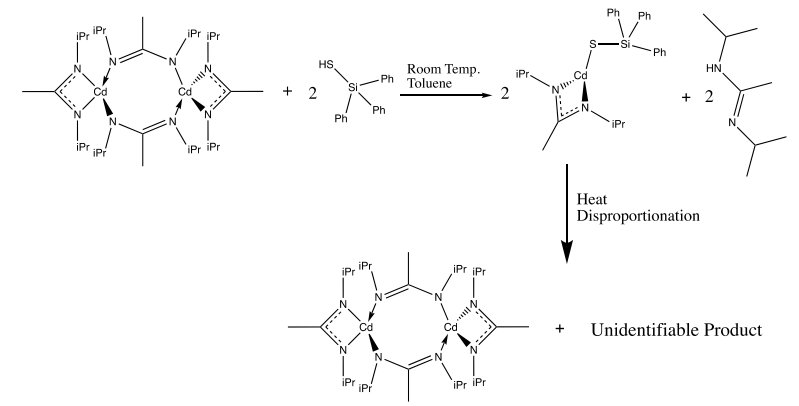

Scheme S1. 4 was observed to disproportionate into 1 and an unidentifiable product during sublimation at $150{ }^{\circ} \mathrm{C}$ (50 mTorr). 
Amidinate Environments in Room Temperature ${ }^{1}$ HNMR for 1-3

Cadmium bis- $N, N$-diisopropylacetamidinate - 1



Figure S1. The room temperature ${ }^{1} \mathrm{HNMR}$ spectrum for $\mathbf{1}$ shows coalescing peaks due to the presence of a monomer/dimer equilibrium. Figure 2 in the main text is a variable temperature ${ }^{1} \mathrm{HNMR}$ that shows the peaks of the dimer at the slow exchange limit and the monomer at the fast exchange limit. 


\section{Cadmium bis- $N, N$-diisopropyltertertiarybutylamidinate - $\mathbf{2}$}

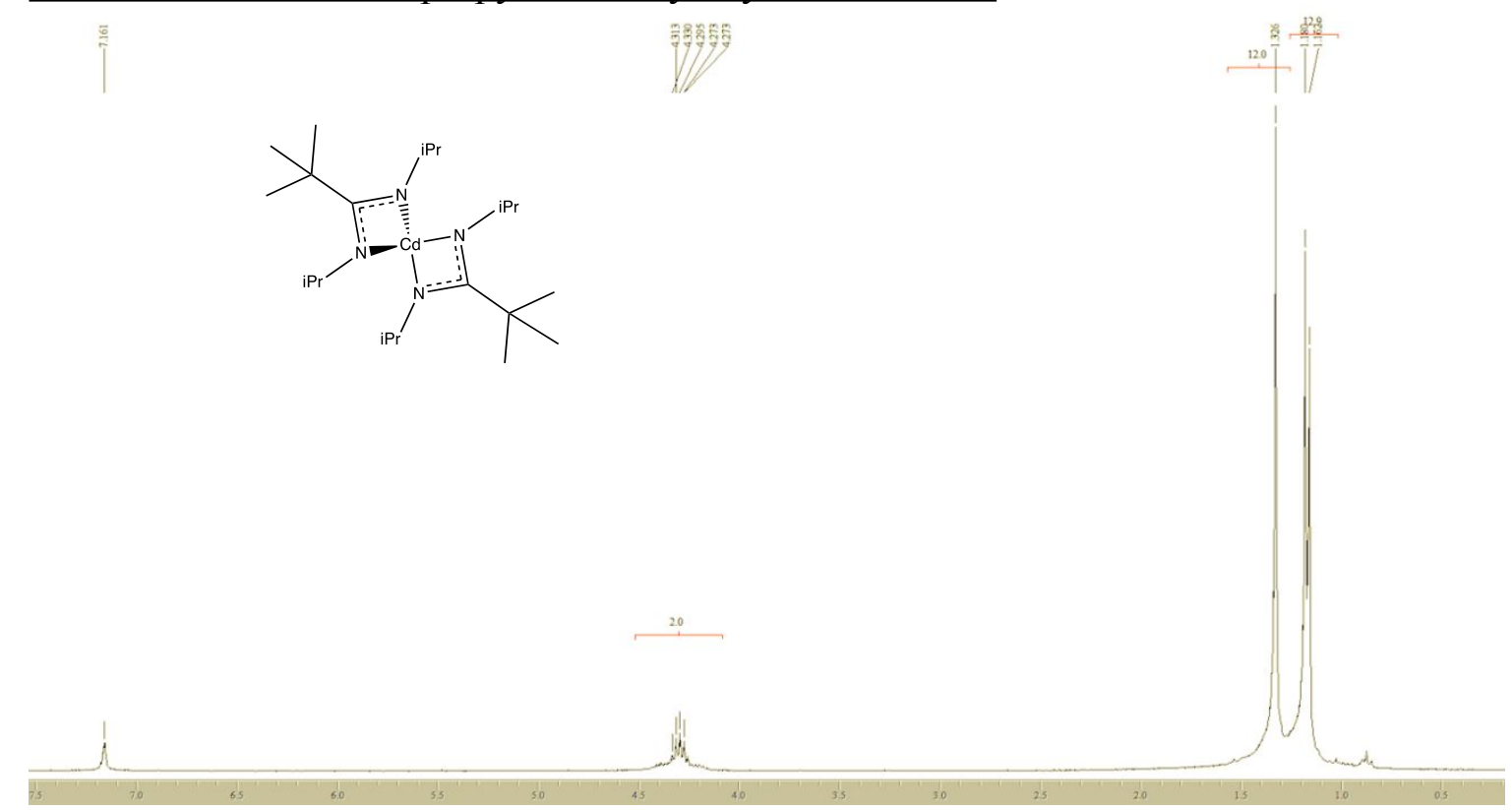

Figure S2. At room temperature there is only one set of clearly defined peaks corresponding to the amidinate ligand. The single set of peaks is consistent with monomeric solution phase 2. 
Cadmium bis- $N, N$-diisopropylbutylamidinate $-\mathbf{3}$

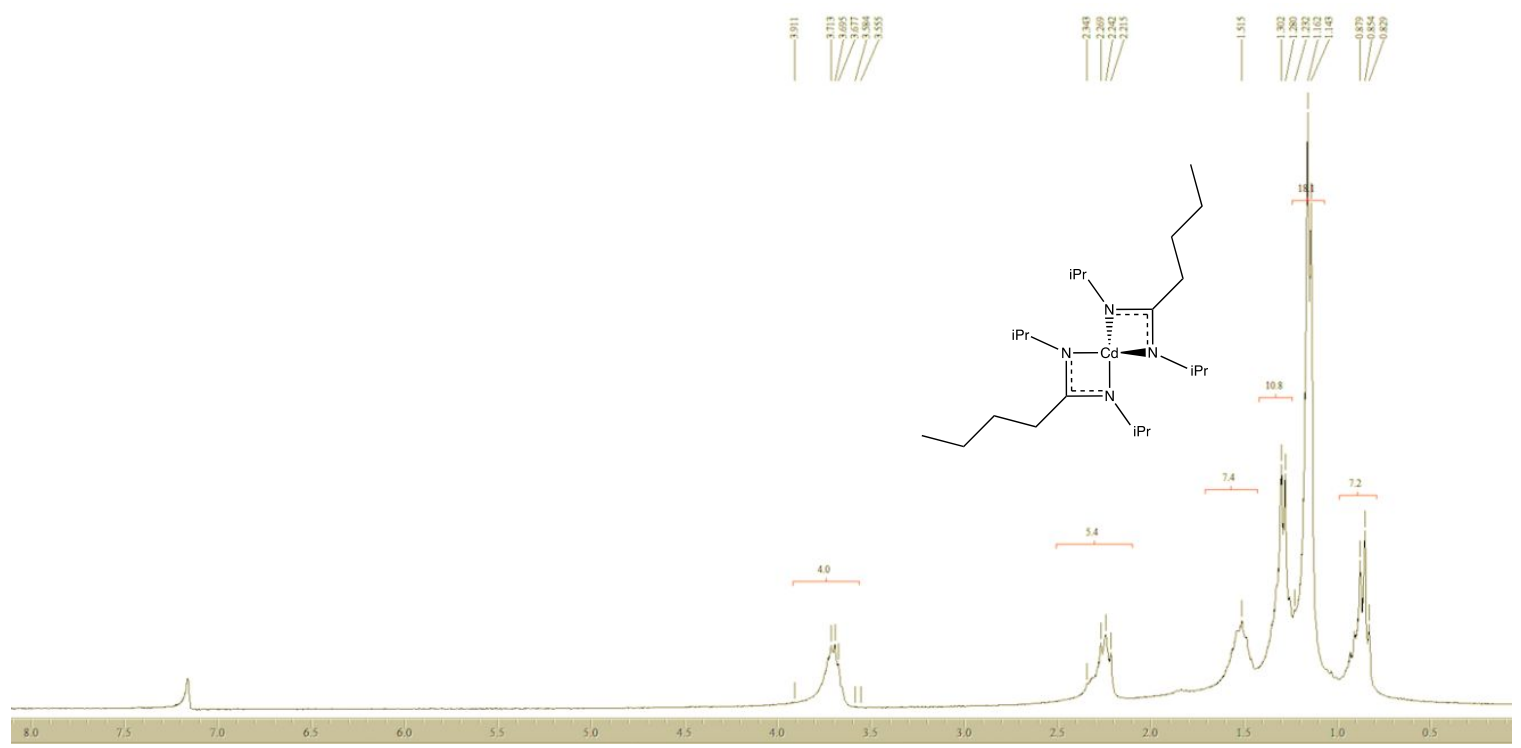

Figure S3. The six peaks in the above room temperature ${ }^{1} \mathrm{HNMR}$ spectrum correspond to the $\operatorname{six}{ }^{1} \mathrm{H}$ environments in $\mathbf{3}$. There is only one set of amidinate peaks present, which is consistent with monomeric solution phase $\mathbf{3}$.

\section{H/D Exchange:}
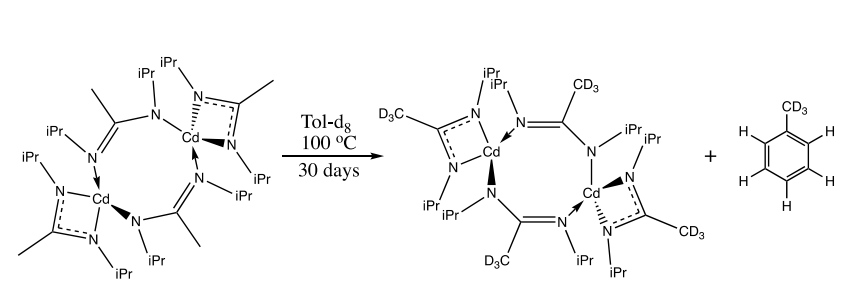

Aryl $\mathrm{C}_{7} \mathrm{D}_{8}$ proton residue peaks increase

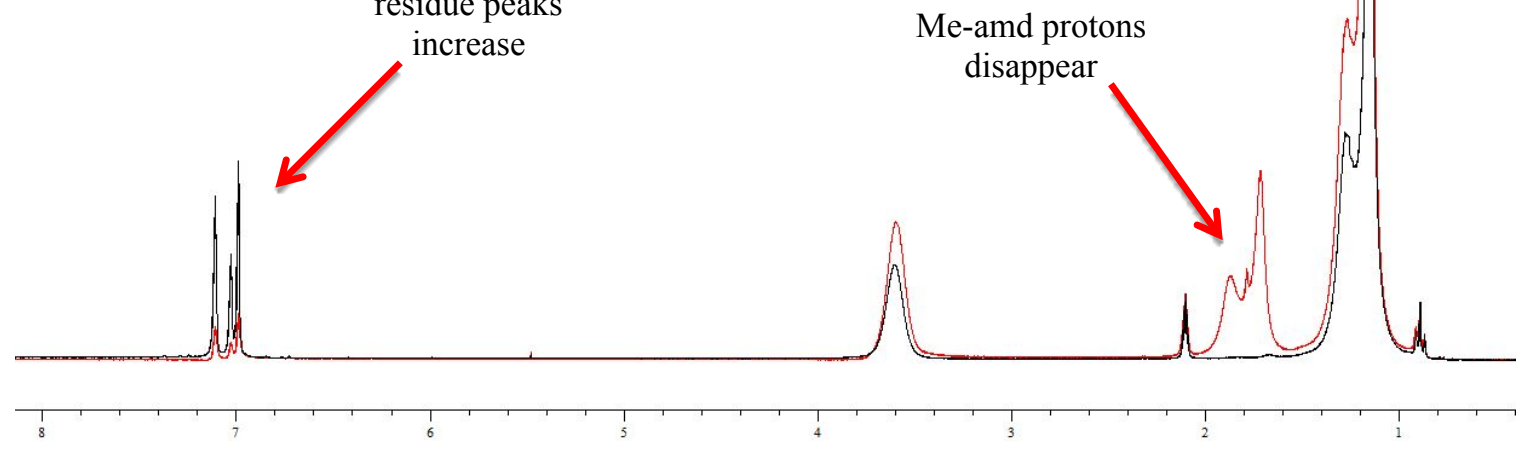

Figure S4. The red spectrum above shows ${ }^{1} \mathrm{HNMR}$ of 1 in tol- $d_{8}$. The black spectrum shows 1 after heating to $100^{\circ} \mathrm{C}$ for 30 days. The sample of 1 was sealed in a J. Young tube during the course of heating to ensure that any changes observed are the result of the heating process. The acetamidinate methyl peak disappears completely over time and the isopropyl peaks of 1 lessen. The tol- $d_{8}$ aryl solvent residue peaks increase and the tol- $d_{8}$ methyl peak (2.1 ppm) does not change. These data are interpreted as an H/D exchange between the aryl tol- $d_{8}$ deuterium atoms and the protons of $\mathbf{1}$, primarily of the methyl- 
amidinate protons. The deuterium atoms of the $-\mathrm{CD}_{3}$ peak of tol- $d_{8}$ do not participate in $\mathrm{H} / \mathrm{D}$ exchange as the peak remains unchanged over the course of the experiment.

\section{Thermal Decomposition of 2}

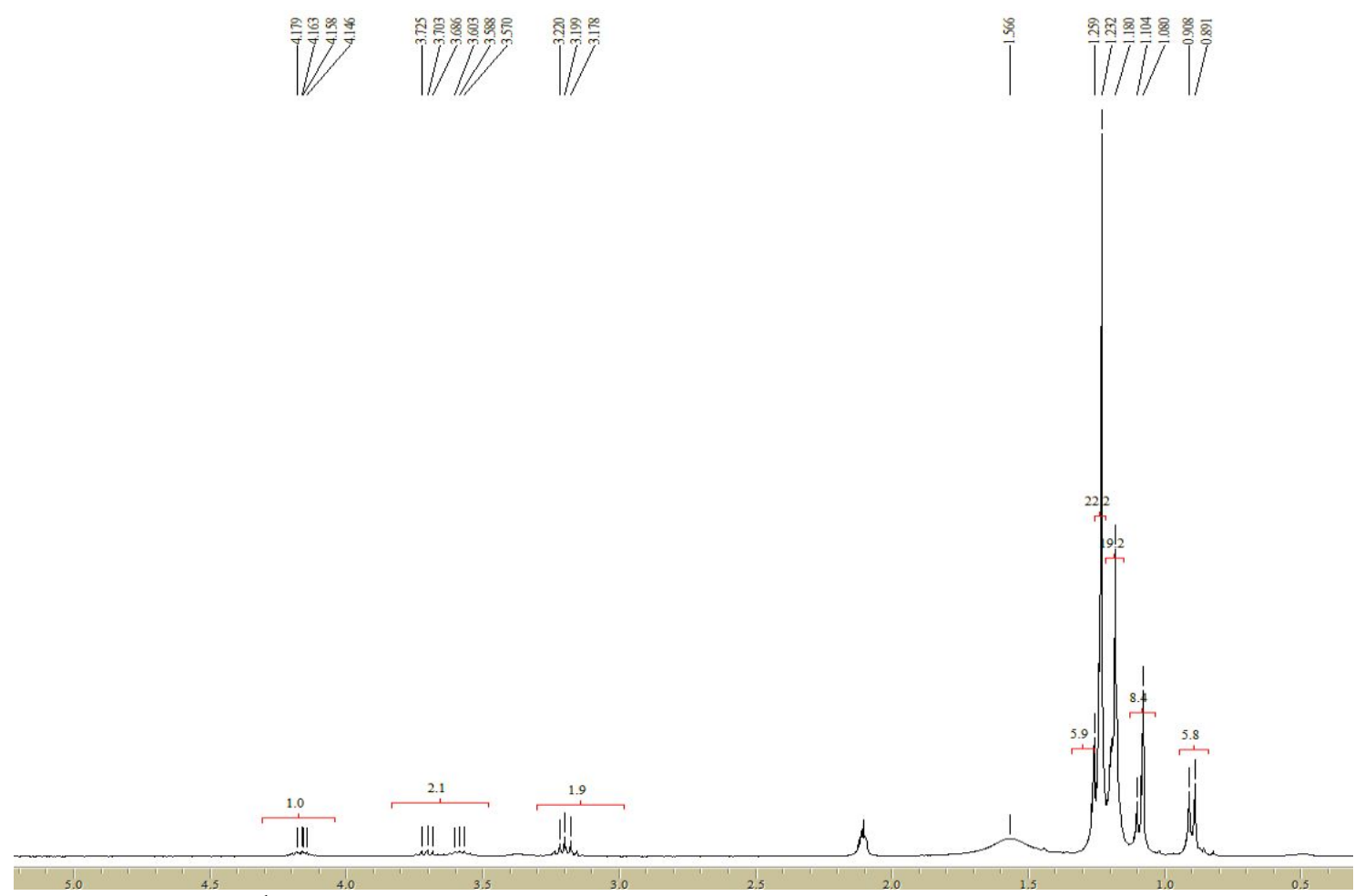

Figure S5. The ${ }^{1} \mathrm{HNMR}$ spectrum above was taken after heating 2 to $100{ }^{\circ} \mathrm{C}$ in a J. Young tube for 24 hours. Subsequent heating did not change the spectrum. Notably, no peaks from the original spectrum $\mathbf{2}$ can be seen. Analysis of decomposed $\mathbf{2}$ shows four new $i \mathrm{Pr}-\mathrm{CH}$ multiplets, four new $i \mathrm{Pr}-\mathrm{CH}_{3}$ doublets, one $t \mathrm{Bu}$ singlet, and a broad peak at $1.5 \mathrm{ppm}$. Gordon, et al. has shown that amidinates can eliminate to yield hydrazine byproducts in ruthenium tris- $N, N$ '-diisopropylacetamidinate. A reductive elimination product is suspected in this case as decomposition is accompanied by the formation of a metallic solid in the NMR tube, presumably $\mathrm{Cd}^{0}$. The appearance of four new $i \mathrm{Pr}$ environments suggests an asymmetric product with four distinct $i \operatorname{Pr}$ proton environments. The appearance of a broad peak at $1.5 \mathrm{ppm}$ could possibly be due to the formation of an amine group during decomposition. It is not possible to reconstruct the decomposition product from its ${ }^{1} \mathrm{HNMR}$ spectrum alone, and these observations clearly show $\mathbf{2}$ to be unstable at $100{ }^{\circ} \mathrm{C}$. 
NMR Spectra of 4 and 5

$\underline{\mathrm{Cd}(\mathrm{amd})\left(\mathrm{SSiPh}_{3}\right)-\mathbf{4}}$

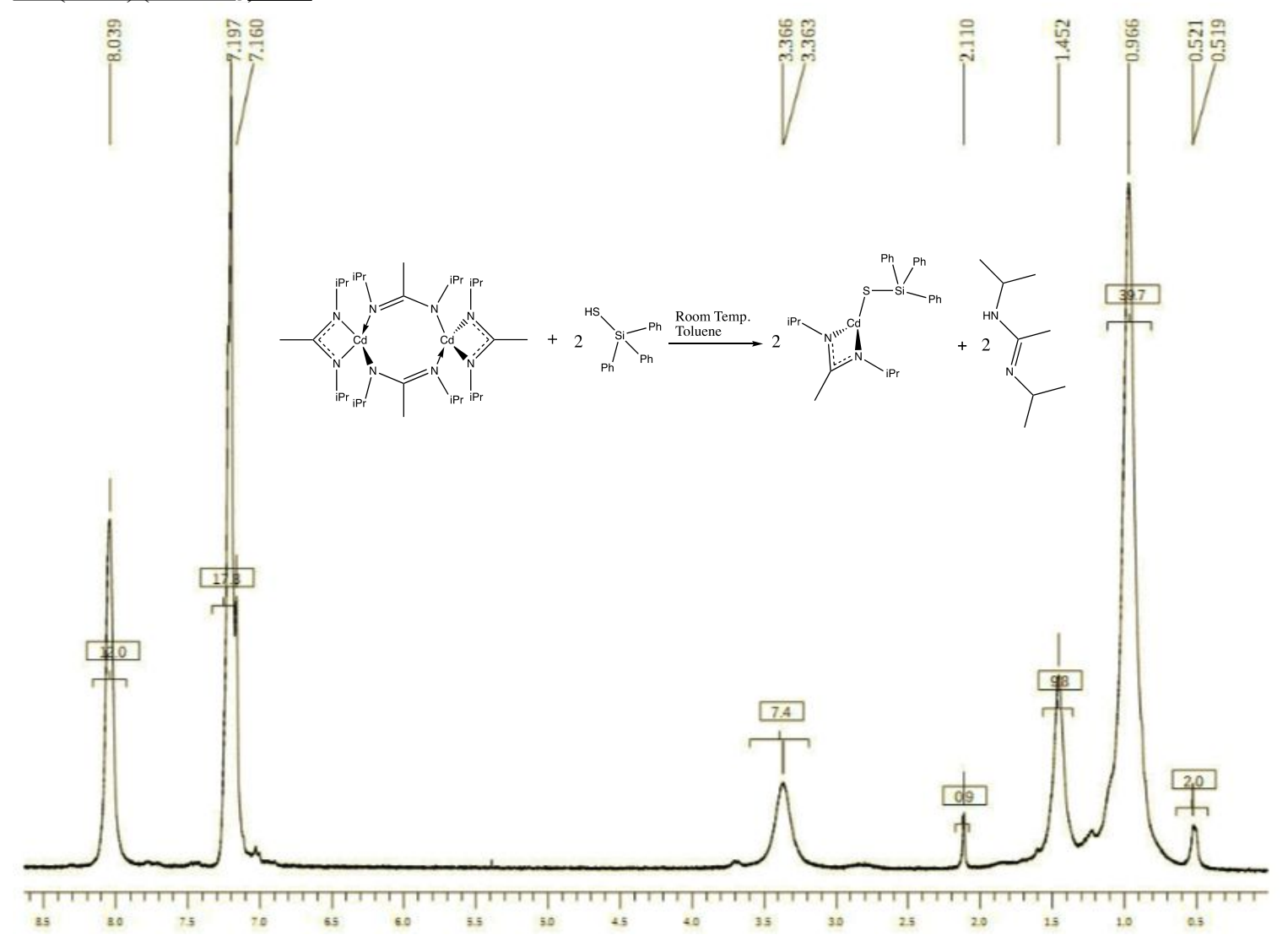

Figure S6. ${ }^{1} \mathrm{HNMR}$ analysis of the reaction of $\mathbf{1}$ and triphenylsilylthiol shows a peak shift and different shape to the set of amidinate peaks. The phenyl peaks integrate as expected, which indicates the formation of a heteroleptic compound 4. 


\section{$\underline{\mathrm{Cd}\left(\mathrm{SSiPh}_{3}\right)(\mathrm{TMEDA})-\mathbf{5}}$}

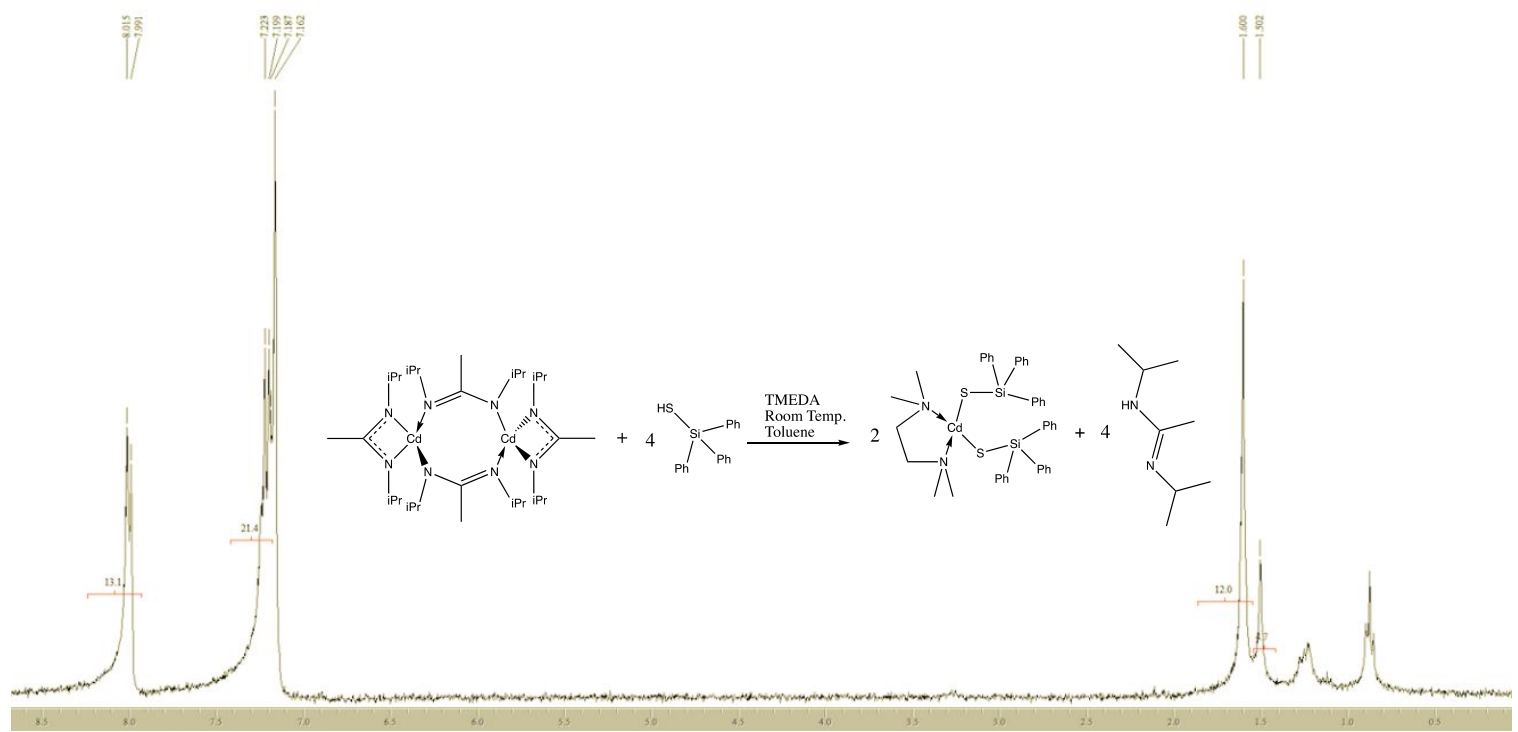

Figure S7. 5 has poor solubility in $\mathrm{C}_{6} \mathrm{D}_{6}$, but there is enough solubility to observe the expected product. Peaks at 1.5 and $1.6 \mathrm{ppm}$ correspond to the TMEDA proton environments and the peaks at 7.2 and $8.0 \mathrm{ppm}$ correspond to the phenyl protons. 
Table S1. Crystallographic details for 1:

Atomic coordinates $\left(\mathrm{x} 10^{4}\right)$ and equivalent isotropic displacement parameters $\left(\AA^{2} \times 10^{3}\right)$

For $1, \mathrm{Cd}[\mathrm{iPrNC}(\mathrm{Me}) \mathrm{NiPr}] 2$. $\mathrm{U}(\mathrm{eq})$ is defined as one third of the trace of the orthogonalized $\mathrm{U}^{\mathrm{ij}}$ tensor.

\begin{tabular}{|c|c|c|c|c|}
\hline & $\mathrm{x}$ & $\mathrm{y}$ & $\mathrm{z}$ & $\mathrm{U}(\mathrm{eq})$ \\
\hline $\mathrm{Cd}(1)$ & $4293(1)$ & 2773(1) & $2088(1)$ & $20(1)$ \\
\hline $\mathrm{N}(1)$ & $3398(1)$ & 1792(2) & $1958(1)$ & $24(1)$ \\
\hline $\mathrm{N}(2)$ & $3420(1)$ & $3683(2)$ & $1262(1)$ & $24(1)$ \\
\hline $\mathrm{N}(3)$ & $4797(1)$ & $1808(2)$ & $1389(1)$ & $24(1)$ \\
\hline $\mathrm{N}(4)$ & $5375(1)$ & $3735(2)$ & $1724(1)$ & $27(1)$ \\
\hline$C(1)$ & $3121(1)$ & $747(3)$ & $2302(1)$ & $30(1)$ \\
\hline$C(2)$ & $3563(1)$ & $395(3)$ & $3106(2)$ & $48(1)$ \\
\hline$C(3)$ & 2951(1) & $-558(3)$ & $1803(2)$ & $45(1)$ \\
\hline$C(4)$ & $3093(1)$ & $2730(2)$ & $1454(1)$ & $23(1)$ \\
\hline$C(5)$ & $2413(1)$ & 2721(3) & $1119(2)$ & $35(1)$ \\
\hline$C(6)$ & $3170(1)$ & $4759(3)$ & $690(1)$ & $31(1)$ \\
\hline$C(7)$ & $3648(1)$ & $5208(3)$ & $359(2)$ & $47(1)$ \\
\hline$C(8)$ & $4567(1)$ & $583(3)$ & $899(1)$ & $30(1)$ \\
\hline$C(9)$ & $4547(1)$ & $-624(3)$ & $1418(2)$ & $40(1)$ \\
\hline$C(10)$ & $3946(1)$ & $852(3)$ & $297(2)$ & $41(1)$ \\
\hline $\mathrm{C}(11)$ & $5124(1)$ & $2763(2)$ & $1200(1)$ & $26(1)$ \\
\hline$C(12)$ & $5209(1)$ & $2735(3)$ & $413(1)$ & $40(1)$ \\
\hline$C(13)$ & $5673(1)$ & $4946(3)$ & $1538(2)$ & $38(1)$ \\
\hline$C(14)$ & $6345(1)$ & $4705(3)$ & $1725(2)$ & $42(1)$ \\
\hline$C(15)$ & $2946(2)$ & $6005(3)$ & $1033(2)$ & $53(1)$ \\
\hline$C(16)$ & $5566(2)$ & $6184(3)$ & 1983(2) & $59(1)$ \\
\hline
\end{tabular}


Bond lengths $[\AA]$ and angles $\left[{ }^{\circ}\right]$ for $\mathrm{Cd}[\mathrm{iPrNC}(\mathrm{Me}) \mathrm{NiPr}] 2$.

\begin{tabular}{|c|c|}
\hline $\mathrm{Cd}(1)-\mathrm{N}(3)$ & $2.2311(17)$ \\
\hline $\mathrm{Cd}(1)-\mathrm{N}(4 \mathrm{~A})$ & $2.2422(18)$ \\
\hline $\mathrm{Cd}(1)-\mathrm{N}(1)$ & $2.2671(17)$ \\
\hline $\mathrm{Cd}(1)-\mathrm{N}(2)$ & $2.2838(17)$ \\
\hline $\mathrm{Cd}(1)-\mathrm{C}(4)$ & $2.689(2)$ \\
\hline $\mathrm{Cd}(1)-\mathrm{Cd}(1 \mathrm{~A})$ & $3.1798(3)$ \\
\hline $\mathrm{N}(1)-\mathrm{C}(4)$ & $1.318(3)$ \\
\hline $\mathrm{N}(1)-\mathrm{C}(1)$ & $1.458(3)$ \\
\hline $\mathrm{N}(2)-\mathrm{C}(4)$ & $1.326(3)$ \\
\hline $\mathrm{N}(2)-\mathrm{C}(6)$ & $1.451(3)$ \\
\hline $\mathrm{N}(3)-\mathrm{C}(11)$ & $1.327(3)$ \\
\hline $\mathrm{N}(3)-\mathrm{C}(8)$ & $1.471(3)$ \\
\hline $\mathrm{N}(4)-\mathrm{C}(11)$ & $1.327(3)$ \\
\hline $\mathrm{N}(4)-\mathrm{C}(13)$ & $1.466(3)$ \\
\hline $\mathrm{N}(4)-\mathrm{Cd}(1 \mathrm{~A})$ & $2.2422(18)$ \\
\hline $\mathrm{C}(1)-\mathrm{C}(2)$ & $1.524(3)$ \\
\hline $\mathrm{C}(1)-\mathrm{C}(3)$ & $1.527(4)$ \\
\hline $\mathrm{C}(4)-\mathrm{C}(5)$ & $1.520(3)$ \\
\hline $\mathrm{C}(6)-\mathrm{C}(7)$ & $1.522(4)$ \\
\hline$C(6)-C(15)$ & $1.533(4)$ \\
\hline $\mathrm{C}(8)-\mathrm{C}(9)$ & $1.514(4)$ \\
\hline$C(8)-C(10)$ & $1.531(3)$ \\
\hline $\mathrm{C}(11)-\mathrm{C}(12)$ & $1.518(3)$ \\
\hline$C(13)-C(16)$ & $1.515(4)$ \\
\hline$C(13)-C(14)$ & $1.532(4)$ \\
\hline $\mathrm{N}(3)-\mathrm{Cd}(1)-\mathrm{N}(4 \mathrm{~A})$ & $130.31(6)$ \\
\hline $\mathrm{N}(3)-\mathrm{Cd}(1)-\mathrm{N}(1)$ & $115.73(7)$ \\
\hline $\mathrm{N}(4 \mathrm{~A})-\mathrm{Cd}(1)-\mathrm{N}(1)$ & $106.35(7)$ \\
\hline $\mathrm{N}(3)-\mathrm{Cd}(1)-\mathrm{N}(2)$ & $109.12(6)$ \\
\hline $\mathrm{N}(4 \mathrm{~A})-\mathrm{Cd}(1)-\mathrm{N}(2)$ & $114.82(7)$ \\
\hline $\mathrm{N}(1)-\mathrm{Cd}(1)-\mathrm{N}(2)$ & $58.82(7)$ \\
\hline $\mathrm{N}(3)-\mathrm{Cd}(1)-\mathrm{C}(4)$ & $116.44(6)$ \\
\hline $\mathrm{N}(4 \mathrm{~A})-\mathrm{Cd}(1)-\mathrm{C}(4)$ & $113.21(6)$ \\
\hline
\end{tabular}




\begin{tabular}{|c|c|}
\hline $\mathrm{N}(1)-\mathrm{Cd}(1)-\mathrm{C}(4)$ & $29.31(7)$ \\
\hline $\mathrm{N}(2)-\mathrm{Cd}(1)-\mathrm{C}(4)$ & $29.52(7)$ \\
\hline $\mathrm{N}(3)-\mathrm{Cd}(1)-\mathrm{Cd}(1 \mathrm{~A})$ & $65.40(5)$ \\
\hline $\mathrm{N}(4 \mathrm{~A})-\mathrm{Cd}(1)-\mathrm{Cd}(1 \mathrm{~A})$ & $64.92(5)$ \\
\hline $\mathrm{N}(1)-\mathrm{Cd}(1)-\mathrm{Cd}(1 \mathrm{~A})$ & $148.49(5)$ \\
\hline $\mathrm{N}(2)-\mathrm{Cd}(1)-\mathrm{Cd}(1 \mathrm{~A})$ & $152.65(5)$ \\
\hline$C(4)-C d(1)-C d(1 A)$ & $177.31(5)$ \\
\hline $\mathrm{C}(4)-\mathrm{N}(1)-\mathrm{C}(1)$ & $123.72(18)$ \\
\hline $\mathrm{C}(4)-\mathrm{N}(1)-\mathrm{Cd}(1)$ & $93.36(13)$ \\
\hline $\mathrm{C}(1)-\mathrm{N}(1)-\mathrm{Cd}(1)$ & $142.12(14)$ \\
\hline $\mathrm{C}(4)-\mathrm{N}(2)-\mathrm{C}(6)$ & $123.64(18)$ \\
\hline $\mathrm{C}(4)-\mathrm{N}(2)-\mathrm{Cd}(1)$ & $92.41(13)$ \\
\hline $\mathrm{C}(6)-\mathrm{N}(2)-\mathrm{Cd}(1)$ & $143.93(15)$ \\
\hline $\mathrm{C}(11)-\mathrm{N}(3)-\mathrm{C}(8)$ & $122.02(19)$ \\
\hline $\mathrm{C}(11)-\mathrm{N}(3)-\mathrm{Cd}(1)$ & $109.63(15)$ \\
\hline $\mathrm{C}(8)-\mathrm{N}(3)-\mathrm{Cd}(1)$ & $121.54(14)$ \\
\hline $\mathrm{C}(11)-\mathrm{N}(4)-\mathrm{C}(13)$ & $122.1(2)$ \\
\hline $\mathrm{C}(11)-\mathrm{N}(4)-\mathrm{Cd}(1 \mathrm{~A})$ & $109.14(14)$ \\
\hline $\mathrm{C}(13)-\mathrm{N}(4)-\mathrm{Cd}(1 \mathrm{~A})$ & $121.06(14)$ \\
\hline $\mathrm{N}(1)-\mathrm{C}(1)-\mathrm{C}(2)$ & $107.81(18)$ \\
\hline $\mathrm{N}(1)-\mathrm{C}(1)-\mathrm{C}(3)$ & $112.4(2)$ \\
\hline $\mathrm{C}(2)-\mathrm{C}(1)-\mathrm{C}(3)$ & $110.3(2)$ \\
\hline $\mathrm{N}(1)-\mathrm{C}(4)-\mathrm{N}(2)$ & $115.38(18)$ \\
\hline $\mathrm{N}(1)-\mathrm{C}(4)-\mathrm{C}(5)$ & $122.1(2)$ \\
\hline $\mathrm{N}(2)-\mathrm{C}(4)-\mathrm{C}(5)$ & $122.5(2)$ \\
\hline $\mathrm{N}(1)-\mathrm{C}(4)-\mathrm{Cd}(1)$ & $57.33(10)$ \\
\hline $\mathrm{N}(2)-\mathrm{C}(4)-\mathrm{Cd}(1)$ & $58.07(10)$ \\
\hline $\mathrm{C}(5)-\mathrm{C}(4)-\mathrm{Cd}(1)$ & $178.21(16)$ \\
\hline $\mathrm{N}(2)-\mathrm{C}(6)-\mathrm{C}(7)$ & $108.2(2)$ \\
\hline $\mathrm{N}(2)-\mathrm{C}(6)-\mathrm{C}(15)$ & $112.4(2)$ \\
\hline$C(7)-C(6)-C(15)$ & $110.5(2)$ \\
\hline $\mathrm{N}(3)-\mathrm{C}(8)-\mathrm{C}(9)$ & $108.80(18)$ \\
\hline $\mathrm{N}(3)-\mathrm{C}(8)-\mathrm{C}(10)$ & $111.55(19)$ \\
\hline C(9)-C(8)-C(10) & $110.6(2)$ \\
\hline $\mathrm{N}(3)-\mathrm{C}(11)-\mathrm{N}(4)$ & 117.61(19) \\
\hline $\mathrm{N}(3)-\mathrm{C}(11)-\mathrm{C}(12)$ & $120.9(2)$ \\
\hline
\end{tabular}




$\begin{array}{ll}\mathrm{N}(4)-\mathrm{C}(11)-\mathrm{C}(12) & 121.5(2) \\ \mathrm{N}(4)-\mathrm{C}(13)-\mathrm{C}(16) & 108.8(2) \\ \mathrm{N}(4)-\mathrm{C}(13)-\mathrm{C}(14) & 112.2(2) \\ \mathrm{C}(16)-\mathrm{C}(13)-\mathrm{C}(14) & 110.7(2)\end{array}$

Symmetry transformations used to generate equivalent atoms:

$\# 1-\mathrm{x}+1, \mathrm{y},-\mathrm{z}+1 / 2$

Anisotropic displacement parameters $\left(\AA^{2} \times 10^{3}\right)$ for $\mathrm{Cd}[\mathrm{iPrNC}(\mathrm{Me}) \mathrm{NiPr}] 22$. The anisotropic displacement factor exponent takes the form: $-2 \square^{2}\left[h^{2} a^{* 2} U^{11}+\ldots+2 h \mathrm{k}^{*} b^{*} U^{12}\right]$

\begin{tabular}{|c|c|c|c|c|c|c|}
\hline & $\mathrm{U}^{11}$ & $\mathrm{U}^{22}$ & $\mathrm{U}^{33}$ & $\mathrm{U}^{23}$ & $\mathrm{U}^{13}$ & $\mathrm{U}^{12}$ \\
\hline $\mathrm{Cd}(1)$ & $17(1)$ & $24(1)$ & $20(1)$ & $-2(1)$ & $7(1)$ & $0(1)$ \\
\hline $\mathrm{N}(1)$ & $21(1)$ & $27(1)$ & $26(1)$ & $-1(1)$ & $9(1)$ & $-4(1)$ \\
\hline $\mathrm{N}(2)$ & $23(1)$ & $26(1)$ & $23(1)$ & $0(1)$ & $8(1)$ & $3(1)$ \\
\hline $\mathrm{N}(3)$ & $22(1)$ & $28(1)$ & $22(1)$ & $-4(1)$ & $9(1)$ & 2(1) \\
\hline $\mathrm{N}(4)$ & $23(1)$ & $30(1)$ & $25(1)$ & $8(1)$ & $6(1)$ & $0(1)$ \\
\hline$C(1)$ & $25(1)$ & $33(1)$ & $34(1)$ & $0(1)$ & $12(1)$ & $-8(1)$ \\
\hline$C(2)$ & $45(2)$ & $55(2)$ & $39(1)$ & $16(1)$ & $9(1)$ & $-17(1)$ \\
\hline$C(3)$ & $47(2)$ & $36(1)$ & $57(2)$ & $-7(1)$ & $21(1)$ & $-16(1)$ \\
\hline$C(4)$ & $16(1)$ & $31(1)$ & $21(1)$ & $-7(1)$ & $3(1)$ & $2(1)$ \\
\hline$C(5)$ & $22(1)$ & $42(1)$ & $37(1)$ & $-5(1)$ & $6(1)$ & $2(1)$ \\
\hline$C(6)$ & $34(1)$ & $32(1)$ & $25(1)$ & $2(1)$ & $7(1)$ & $6(1)$ \\
\hline$C(7)$ & $52(2)$ & $48(2)$ & $42(2)$ & $19(1)$ & $17(1)$ & $-1(1)$ \\
\hline $\mathrm{C}(8)$ & $29(1)$ & $33(1)$ & $26(1)$ & $-9(1)$ & $9(1)$ & $4(1)$ \\
\hline$C(9)$ & $43(1)$ & $29(1)$ & $43(1)$ & $-6(1)$ & $9(1)$ & $0(1)$ \\
\hline$C(10)$ & $37(1)$ & $45(2)$ & $31(1)$ & $-12(1)$ & $0(1)$ & $3(1)$ \\
\hline $\mathrm{C}(11)$ & $22(1)$ & $35(1)$ & $21(1)$ & $7(1)$ & $7(1)$ & $9(1)$ \\
\hline$C(12)$ & $40(1)$ & $57(2)$ & $26(1)$ & $5(1)$ & $16(1)$ & $-1(1)$ \\
\hline $\mathrm{C}(13)$ & $35(1)$ & $37(1)$ & $34(1)$ & $17(1)$ & $5(1)$ & $-4(1)$ \\
\hline$C(14)$ & $35(1)$ & $53(2)$ & $40(1)$ & $15(1)$ & $14(1)$ & $-10(1)$ \\
\hline$C(15)$ & $69(2)$ & $36(2)$ & $50(2)$ & $5(1)$ & $15(2)$ & $23(1)$ \\
\hline$C(16)$ & $50(2)$ & $28(1)$ & $93(3)$ & $11(2)$ & $19(2)$ & $-2(1)$ \\
\hline
\end{tabular}


Hydrogen coordinates ( x $\left.10^{4}\right)$ and isotropic displacement parameters $\left(\AA^{2} \times 10^{3}\right)$ for $\mathrm{Cd}[\mathrm{iPrNC}(\mathrm{Me}) \mathrm{niPr}] 22$.

\begin{tabular}{|c|c|c|c|c|}
\hline & $\mathrm{x}$ & $\mathrm{y}$ & $\mathrm{z}$ & $\mathrm{U}(\mathrm{eq})$ \\
\hline $\mathrm{H}(1)$ & 2752 & 1148 & 2362 & 36 \\
\hline $\mathrm{H}(2 \mathrm{~A})$ & 3938 & 63 & 3057 & 71 \\
\hline $\mathrm{H}(2 \mathrm{~B})$ & 3393 & -328 & 3344 & 71 \\
\hline $\mathrm{H}(2 \mathrm{C})$ & 3642 & 1225 & 3435 & 71 \\
\hline $\mathrm{H}(3 \mathrm{~A})$ & 2681 & -312 & 1280 & 68 \\
\hline $\mathrm{H}(3 \mathrm{~B})$ & 2750 & -1210 & 2043 & 68 \\
\hline $\mathrm{H}(3 \mathrm{C})$ & 3313 & -990 & 1765 & 68 \\
\hline $\mathrm{H}(5 \mathrm{~A})$ & 2272 & 1773 & 972 & 52 \\
\hline $\mathrm{H}(5 \mathrm{~B})$ & 2278 & 3319 & 658 & 52 \\
\hline $\mathrm{H}(5 \mathrm{C})$ & 2251 & 3065 & 1512 & 52 \\
\hline $\mathrm{H}(6)$ & 2825 & 4362 & 257 & 38 \\
\hline $\mathrm{H}(7 \mathrm{~A})$ & 3999 & 5544 & 782 & 71 \\
\hline $\mathrm{H}(7 \mathrm{~B})$ & 3490 & 5951 & -22 & 71 \\
\hline $\mathrm{H}(7 \mathrm{C})$ & 3762 & 4418 & 103 & 71 \\
\hline $\mathrm{H}(8)$ & 4848 & 350 & 617 & 36 \\
\hline $\mathrm{H}(9 \mathrm{~A})$ & 4950 & -810 & 1783 & 60 \\
\hline $\mathrm{H}(9 \mathrm{~B})$ & 4395 & -1447 & 1098 & 60 \\
\hline $\mathrm{H}(9 \mathrm{C})$ & 4282 & -397 & 1711 & 60 \\
\hline $\mathrm{H}(10 \mathrm{~A})$ & 3665 & 1082 & 566 & 61 \\
\hline $\mathrm{H}(10 \mathrm{~B})$ & 3807 & 20 & -21 & 61 \\
\hline $\mathrm{H}(10 \mathrm{C})$ & 3969 & 1626 & -39 & 61 \\
\hline $\mathrm{H}(12 \mathrm{~A})$ & 5425 & 1892 & 370 & 60 \\
\hline $\mathrm{H}(12 \mathrm{~B})$ & 5440 & 3549 & 364 & 60 \\
\hline $\mathrm{H}(12 \mathrm{C})$ & 4817 & 2747 & -2 & 60 \\
\hline $\mathrm{H}(13)$ & 5488 & 5141 & 968 & 45 \\
\hline $\mathrm{H}(14 \mathrm{~A})$ & 6540 & 4595 & 2288 & 64 \\
\hline $\mathrm{H}(14 \mathrm{~B})$ & 6518 & 5501 & 1545 & 64 \\
\hline $\mathrm{H}(14 \mathrm{C})$ & 6405 & 3866 & 1459 & 64 \\
\hline $\mathrm{H}(15 \mathrm{~A})$ & 2644 & 5697 & 1252 & 79 \\
\hline $\mathrm{H}(15 \mathrm{~B})$ & 2768 & 6687 & 621 & 79 \\
\hline
\end{tabular}




\begin{tabular}{lllll}
$\mathrm{H}(15 \mathrm{C})$ & 3282 & 6428 & 1444 & 79 \\
$\mathrm{H}(16 \mathrm{~A})$ & 5135 & 6346 & 1838 & 88 \\
$\mathrm{H}(16 \mathrm{~B})$ & 5757 & 7004 & 1855 & 88 \\
$\mathrm{H}(16 \mathrm{C})$ & 5737 & 6002 & 2544 & 88 \\
\hline
\end{tabular}


Table S2. Crystallographic data for 5:

Atomic coordinates $\left(\mathrm{x} 10^{4}\right)$ and equivalent isotropic displacement parameters $\left(\AA^{2} \times 10^{3}\right)$ for $\mathbf{5}, \mathrm{Cd}(\mathrm{SSiPh} 3) 2(\mathrm{TMEDA})$. $\mathrm{U}(\mathrm{eq})$ is defined as one third of the trace of the orthogonalized $\mathrm{U}^{\mathrm{ij}}$ tensor.

\begin{tabular}{|c|c|c|c|c|}
\hline & $\mathrm{x}$ & $\mathrm{y}$ & $\mathrm{z}$ & $\mathrm{U}(\mathrm{eq})$ \\
\hline $\mathrm{Cd}(1)$ & $908(1)$ & $2810(1)$ & $1855(1)$ & $24(1)$ \\
\hline $\mathrm{N}(1)$ & 1284(1) & $2968(1)$ & $245(1)$ & $31(1)$ \\
\hline $\mathrm{N}(2)$ & $-1338(1)$ & $2678(1)$ & $1846(1)$ & $33(1)$ \\
\hline$S(1)$ & 1254(1) & $4555(1)$ & $1712(1)$ & $28(1)$ \\
\hline$S(2)$ & 1164(1) & $1069(1)$ & $2510(1)$ & $30(1)$ \\
\hline $\operatorname{Si}(1)$ & $857(1)$ & $4696(1)$ & $3057(1)$ & $24(1)$ \\
\hline $\operatorname{Si}(2)$ & $3127(1)$ & 1059(1) & $2574(1)$ & $24(1)$ \\
\hline $\mathrm{C}(1)$ & 2445(2) & $4648(1)$ & $3369(1)$ & $29(1)$ \\
\hline$C(2)$ & 3623(2) & $5204(1)$ & $2732(1)$ & $38(1)$ \\
\hline$C(3)$ & 4813(2) & $5179(2)$ & 2941(2) & $47(1)$ \\
\hline$C(4)$ & 4851(2) & $4600(2)$ & $3792(2)$ & $53(1)$ \\
\hline$C(5)$ & 3699(2) & $4044(2)$ & $4433(2)$ & $57(1)$ \\
\hline$C(6)$ & 2505(2) & $4068(2)$ & $4227(1)$ & $43(1)$ \\
\hline$C(7)$ & $-459(2)$ & $3726(1)$ & $3963(1)$ & $27(1)$ \\
\hline$C(8)$ & $-227(2)$ & $2725(1)$ & $4134(1)$ & $36(1)$ \\
\hline $\mathrm{C}(9)$ & $-1227(2)$ & 1997(1) & $4760(1)$ & $47(1)$ \\
\hline$C(10)$ & $-2461(2)$ & $2250(2)$ & $5244(1)$ & $50(1)$ \\
\hline $\mathrm{C}(11)$ & $-2701(2)$ & $3228(2)$ & $5102(1)$ & $45(1)$ \\
\hline$C(12)$ & $-1712(2)$ & $3965(1)$ & $4465(1)$ & $34(1)$ \\
\hline$C(13)$ & $170(2)$ & $5925(1)$ & $3032(1)$ & $28(1)$ \\
\hline$C(14)$ & $-887(2)$ & $6177(2)$ & $2677(2)$ & $47(1)$ \\
\hline$C(15)$ & $-1489(3)$ & $7046(2)$ & $2708(2)$ & $62(1)$ \\
\hline$C(16)$ & $-1033(3)$ & $7688(2)$ & $3078(2)$ & $55(1)$ \\
\hline$C(17)$ & $-15(2)$ & $7455(1)$ & $3441(2)$ & $49(1)$ \\
\hline$C(18)$ & $588(2)$ & $6578(1)$ & $3419(1)$ & $39(1)$ \\
\hline$C(19)$ & $3144(2)$ & 1096(1) & $3743(1)$ & $28(1)$ \\
\hline$C(20)$ & 2017(2) & $707(1)$ & $4533(1)$ & $36(1)$ \\
\hline$C(21)$ & 2052(2) & $596(2)$ & $5422(1)$ & $48(1)$ \\
\hline$C(22)$ & $3214(3)$ & $867(2)$ & $5538(2)$ & $55(1)$ \\
\hline$C(23)$ & 4339(2) & $1276(2)$ & $4773(2)$ & $62(1)$ \\
\hline
\end{tabular}




\begin{tabular}{|c|c|c|c|c|}
\hline$C(24)$ & $4297(2)$ & $1396(2)$ & 3882(1) & $47(1)$ \\
\hline$C(25)$ & $4287(1)$ & 2101(1) & 1636(1) & $27(1)$ \\
\hline$C(26)$ & $4319(2)$ & $3049(1)$ & $1737(1)$ & $33(1)$ \\
\hline$C(27)$ & $5080(2)$ & $3844(1)$ & 1013(1) & $41(1)$ \\
\hline$C(28)$ & $5833(2)$ & $3709(1)$ & $173(1)$ & $42(1)$ \\
\hline $\mathrm{C}(29)$ & $5831(2)$ & $2778(2)$ & $60(1)$ & $42(1)$ \\
\hline$C(30)$ & $5064(2)$ & 1981(1) & 781(1) & $35(1)$ \\
\hline $\mathrm{C}(31)$ & $3794(2)$ & $-141(1)$ & $2423(1)$ & $30(1)$ \\
\hline$C(32)$ & $4965(2)$ & $-432(1)$ & $2608(2)$ & $49(1)$ \\
\hline$C(33)$ & $5450(2)$ & $-1327(2)$ & $2525(2)$ & $63(1)$ \\
\hline$C(34)$ & $4775(2)$ & $-1950(2)$ & $2259(2)$ & $57(1)$ \\
\hline$C(35)$ & $3579(3)$ & $-1708(2)$ & $2125(2)$ & $62(1)$ \\
\hline$C(36)$ & $3095(2)$ & $-810(2)$ & $2202(2)$ & $52(1)$ \\
\hline$C(37)$ & $-2209(2)$ & $3297(2)$ & $2354(2)$ & $49(1)$ \\
\hline$C(38)$ & $-1946(2)$ & $1654(2)$ & $2247(2)$ & $52(1)$ \\
\hline $\mathrm{C}(39)$ & $-1200(4)$ & $3090(4)$ & $851(3)$ & $51(1)$ \\
\hline$C(40)$ & $-57(3)$ & $2672(4)$ & $228(2)$ & $45(1)$ \\
\hline$C(40 A)$ & $-37(15)$ & $3250(20)$ & $158(11)$ & $46(5)$ \\
\hline$C(39 A)$ & $-1020(20)$ & $2670(30)$ & $841(19)$ & $66(8)$ \\
\hline $\mathrm{C}(41)$ & $1819(3)$ & $3962(2)$ & $-342(1)$ & $61(1)$ \\
\hline$C(42)$ & 2199(3) & $2267(2)$ & $-88(2)$ & $55(1)$ \\
\hline $\mathrm{C}(1 \mathrm{~T})$ & $11674(7)$ & $-326(6)$ & $9775(8)$ & $48(2)$ \\
\hline $\mathrm{C}(2 \mathrm{~T})$ & $11294(7)$ & $183(6)$ & $9019(6)$ & $52(2)$ \\
\hline $\mathrm{C}(3 \mathrm{~T})$ & $9967(8)$ & $397(8)$ & $9151(6)$ & $41(2)$ \\
\hline $\mathrm{C}(4 \mathrm{~T})$ & $9020(7)$ & $103(11)$ & $10039(7)$ & $39(2)$ \\
\hline $\mathrm{C}(5 \mathrm{~T})$ & $9400(11)$ & $-406(12)$ & $10795(6)$ & $46(3)$ \\
\hline $\mathrm{C}(6 \mathrm{~T})$ & $10728(12)$ & $-621(8)$ & $10663(7)$ & $49(2)$ \\
\hline $\mathrm{C}(7 \mathrm{~T})$ & 7661(9) & $418(7)$ & $10117(7)$ & $43(2)$ \\
\hline $\mathrm{C}(1 \mathrm{~S})$ & $10633(10)$ & $299(15)$ & $9140(8)$ & $50(5)$ \\
\hline $\mathrm{C}(2 \mathrm{~S})$ & $9460(11)$ & $673(10)$ & $9015(5)$ & $54(3)$ \\
\hline $\mathrm{C}(3 \mathrm{~S})$ & $8305(8)$ & $564(6)$ & $9780(7)$ & $45(2)$ \\
\hline $\mathrm{C}(4 \mathrm{~S})$ & $8324(10)$ & $82(7)$ & $10669(6)$ & $73(4)$ \\
\hline$C(5 S)$ & $9497(14)$ & $-292(11)$ & 10794(7) & $69(6)$ \\
\hline$C(6 S)$ & $10652(12)$ & $-184(15)$ & $10029(10)$ & $67(3)$ \\
\hline
\end{tabular}


Bond lengths $[\AA]$ and angles $\left[^{\circ}\right]$ for $\mathrm{Cd}(\mathrm{SSiPh} 3) 2(\mathrm{TMEDA})$.

\begin{tabular}{|c|c|}
\hline $\mathrm{Cd}(1)-\mathrm{N}(2)$ & $2.3803(13)$ \\
\hline $\mathrm{Cd}(1)-\mathrm{N}(1)$ & $2.4035(13)$ \\
\hline $\mathrm{Cd}(1)-\mathrm{S}(1)$ & $2.4431(4)$ \\
\hline $\mathrm{Cd}(1)-\mathrm{S}(2)$ & $2.4435(4)$ \\
\hline $\mathrm{N}(1)-\mathrm{C}(41)$ & $1.453(2)$ \\
\hline $\mathrm{N}(1)-\mathrm{C}(42)$ & $1.466(2)$ \\
\hline $\mathrm{N}(1)-\mathrm{C}(40 \mathrm{~A})$ & $1.481(14)$ \\
\hline $\mathrm{N}(1)-\mathrm{C}(40)$ & $1.489(3)$ \\
\hline $\mathrm{N}(2)-\mathrm{C}(38)$ & $1.471(2)$ \\
\hline $\mathrm{N}(2)-\mathrm{C}(37)$ & $1.475(2)$ \\
\hline $\mathrm{N}(2)-\mathrm{C}(39)$ & $1.481(4)$ \\
\hline $\mathrm{N}(2)-\mathrm{C}(39 \mathrm{~A})$ & $1.53(3)$ \\
\hline $\mathrm{S}(1)-\operatorname{Si}(1)$ & $2.1103(6)$ \\
\hline $\mathrm{S}(2)-\operatorname{Si}(2)$ & $2.1029(5)$ \\
\hline $\mathrm{Si}(1)-\mathrm{C}(13)$ & $1.8773(15)$ \\
\hline $\operatorname{Si}(1)-C(7)$ & $1.8785(15)$ \\
\hline $\operatorname{Si}(1)-C(1)$ & $1.8856(16)$ \\
\hline $\mathrm{Si}(2)-\mathrm{C}(25)$ & $1.8778(15)$ \\
\hline $\mathrm{Si}(2)-\mathrm{C}(19)$ & $1.8782(16)$ \\
\hline $\mathrm{Si}(2)-\mathrm{C}(31)$ & $1.8847(16)$ \\
\hline$C(1)-C(6)$ & $1.398(2)$ \\
\hline $\mathrm{C}(1)-\mathrm{C}(2)$ & $1.398(2)$ \\
\hline $\mathrm{C}(2)-\mathrm{C}(3)$ & $1.393(3)$ \\
\hline$C(3)-C(4)$ & $1.381(3)$ \\
\hline$C(4)-C(5)$ & $1.381(3)$ \\
\hline$C(5)-C(6)$ & $1.396(3)$ \\
\hline$C(7)-C(8)$ & $1.402(2)$ \\
\hline$C(7)-C(12)$ & $1.403(2)$ \\
\hline $\mathrm{C}(8)-\mathrm{C}(9)$ & $1.392(2)$ \\
\hline$C(9)-C(10)$ & $1.386(3)$ \\
\hline$C(10)-C(11)$ & $1.376(3)$ \\
\hline$C(11)-C(12)$ & $1.398(2)$ \\
\hline$C(13)-C(18)$ & $1.392(2)$ \\
\hline$C(13)-C(14)$ & $1.402(2)$ \\
\hline
\end{tabular}




\begin{tabular}{|c|c|}
\hline$C(14)-C(15)$ & $1.387(3)$ \\
\hline$C(15)-C(16)$ & $1.380(3)$ \\
\hline$C(16)-C(17)$ & $1.368(3)$ \\
\hline$C(17)-C(18)$ & $1.395(3)$ \\
\hline$C(19)-C(24)$ & $1.394(2)$ \\
\hline$C(19)-C(20)$ & $1.399(2)$ \\
\hline$C(20)-C(21)$ & $1.387(3)$ \\
\hline$C(21)-C(22)$ & $1.370(3)$ \\
\hline$C(22)-C(23)$ & $1.383(4)$ \\
\hline$C(23)-C(24)$ & $1.392(3)$ \\
\hline$C(25)-C(26)$ & $1.401(2)$ \\
\hline$C(25)-C(30)$ & $1.401(2)$ \\
\hline$C(26)-C(27)$ & $1.390(2)$ \\
\hline$C(27)-C(28)$ & $1.382(3)$ \\
\hline$C(28)-C(29)$ & $1.383(3)$ \\
\hline$C(29)-C(30)$ & $1.391(2)$ \\
\hline$C(31)-C(32)$ & $1.390(2)$ \\
\hline$C(31)-C(36)$ & $1.392(3)$ \\
\hline $\mathrm{C}(32)-\mathrm{C}(33)$ & $1.389(3)$ \\
\hline$C(33)-C(34)$ & $1.374(4)$ \\
\hline C(34)-C(35) & $1.368(4)$ \\
\hline$C(35)-C(36)$ & $1.391(3)$ \\
\hline C(39)-C(40) & $1.529(7)$ \\
\hline$C(40 A)-C(39 A)$ & $1.31(4)$ \\
\hline $\mathrm{C}(1 \mathrm{~T})-\mathrm{C}(2 \mathrm{~T})$ & 1.3900 \\
\hline $\mathrm{C}(1 \mathrm{~T})-\mathrm{C}(6 \mathrm{~T})$ & 1.3900 \\
\hline $\mathrm{C}(2 \mathrm{~T})-\mathrm{C}(3 \mathrm{~T})$ & 1.3900 \\
\hline $\mathrm{C}(3 \mathrm{~T})-\mathrm{C}(4 \mathrm{~T})$ & 1.3900 \\
\hline $\mathrm{C}(4 \mathrm{~T})-\mathrm{C}(5 \mathrm{~T})$ & 1.3900 \\
\hline $\mathrm{C}(4 \mathrm{~T})-\mathrm{C}(7 \mathrm{~T})$ & $1.474(9)$ \\
\hline $\mathrm{C}(5 \mathrm{~T})-\mathrm{C}(6 \mathrm{~T})$ & 1.3900 \\
\hline $\mathrm{C}(1 \mathrm{~S})-\mathrm{C}(2 \mathrm{~S})$ & 1.3900 \\
\hline $\mathrm{C}(1 \mathrm{~S})-\mathrm{C}(6 \mathrm{~S})$ & 1.3900 \\
\hline$C(2 S)-C(3 S)$ & 1.3900 \\
\hline C(3S)-C(4S) & 1.3900 \\
\hline $\mathrm{C}(4 \mathrm{~S})-\mathrm{C}(5 \mathrm{~S})$ & 1.3900 \\
\hline
\end{tabular}




\begin{tabular}{|c|c|}
\hline $\mathrm{C}(5 \mathrm{~S})-\mathrm{C}(6 \mathrm{~S})$ & 1.3900 \\
\hline $\mathrm{N}(2)-\mathrm{Cd}(1)-\mathrm{N}(1)$ & $78.74(5)$ \\
\hline $\mathrm{N}(2)-\mathrm{Cd}(1)-\mathrm{S}(1)$ & $102.07(4)$ \\
\hline $\mathrm{N}(1)-\mathrm{Cd}(1)-\mathrm{S}(1)$ & $98.31(3)$ \\
\hline $\mathrm{N}(2)-\mathrm{Cd}(1)-\mathrm{S}(2)$ & $99.51(4)$ \\
\hline $\mathrm{N}(1)-\mathrm{Cd}(1)-\mathrm{S}(2)$ & $103.25(4)$ \\
\hline$S(1)-C d(1)-S(2)$ & $152.001(14)$ \\
\hline $\mathrm{C}(41)-\mathrm{N}(1)-\mathrm{C}(42)$ & $108.92(17)$ \\
\hline $\mathrm{C}(41)-\mathrm{N}(1)-\mathrm{C}(40 \mathrm{~A})$ & $87.0(11)$ \\
\hline $\mathrm{C}(42)-\mathrm{N}(1)-\mathrm{C}(40 \mathrm{~A})$ & $132.7(12)$ \\
\hline $\mathrm{C}(41)-\mathrm{N}(1)-\mathrm{C}(40)$ & $113.8(2)$ \\
\hline $\mathrm{C}(42)-\mathrm{N}(1)-\mathrm{C}(40)$ & $106.5(2)$ \\
\hline $\mathrm{C}(40 \mathrm{~A})-\mathrm{N}(1)-\mathrm{C}(40)$ & $30.9(11)$ \\
\hline $\mathrm{C}(41)-\mathrm{N}(1)-\mathrm{Cd}(1)$ & $111.22(11)$ \\
\hline $\mathrm{C}(42)-\mathrm{N}(1)-\mathrm{Cd}(1)$ & $112.39(11)$ \\
\hline $\mathrm{C}(40 \mathrm{~A})-\mathrm{N}(1)-\mathrm{Cd}(1)$ & $101.6(7)$ \\
\hline $\mathrm{C}(40)-\mathrm{N}(1)-\mathrm{Cd}(1)$ & $103.98(12)$ \\
\hline $\mathrm{C}(38)-\mathrm{N}(2)-\mathrm{C}(37)$ & $108.61(15)$ \\
\hline $\mathrm{C}(38)-\mathrm{N}(2)-\mathrm{C}(39)$ & $112.7(2)$ \\
\hline $\mathrm{C}(37)-\mathrm{N}(2)-\mathrm{C}(39)$ & $107.5(2)$ \\
\hline $\mathrm{C}(38)-\mathrm{N}(2)-\mathrm{C}(39 \mathrm{~A})$ & $94.1(16)$ \\
\hline $\mathrm{C}(37)-\mathrm{N}(2)-\mathrm{C}(39 \mathrm{~A})$ & $130.3(13)$ \\
\hline $\mathrm{C}(39)-\mathrm{N}(2)-\mathrm{C}(39 \mathrm{~A})$ & $24.2(14)$ \\
\hline $\mathrm{C}(38)-\mathrm{N}(2)-\mathrm{Cd}(1)$ & $112.03(11)$ \\
\hline $\mathrm{C}(37)-\mathrm{N}(2)-\mathrm{Cd}(1)$ & $112.16(11)$ \\
\hline $\mathrm{C}(39)-\mathrm{N}(2)-\mathrm{Cd}(1)$ & $103.65(17)$ \\
\hline $\mathrm{C}(39 \mathrm{~A})-\mathrm{N}(2)-\mathrm{Cd}(1)$ & $98.1(8)$ \\
\hline $\mathrm{Si}(1)-\mathrm{S}(1)-\mathrm{Cd}(1)$ & $108.273(18)$ \\
\hline $\mathrm{Si}(2)-\mathrm{S}(2)-\mathrm{Cd}(1)$ & $104.247(18)$ \\
\hline $\mathrm{C}(13)-\mathrm{Si}(1)-\mathrm{C}(7)$ & $107.48(7)$ \\
\hline $\mathrm{C}(13)-\mathrm{Si}(1)-\mathrm{C}(1)$ & $109.93(7)$ \\
\hline $\mathrm{C}(7)-\mathrm{Si}(1)-\mathrm{C}(1)$ & $109.97(7)$ \\
\hline $\mathrm{C}(13)-\mathrm{Si}(1)-\mathrm{S}(1)$ & $106.48(5)$ \\
\hline $\mathrm{C}(7)-\mathrm{Si}(1)-\mathrm{S}(1)$ & $112.05(5)$ \\
\hline $\mathrm{C}(1)-\mathrm{Si}(1)-\mathrm{S}(1)$ & $110.81(5)$ \\
\hline
\end{tabular}




\begin{tabular}{|c|c|}
\hline $\mathrm{C}(25)-\mathrm{Si}(2)-\mathrm{C}(19)$ & $109.70(7)$ \\
\hline$C(25)-\operatorname{Si}(2)-C(31)$ & $108.52(7)$ \\
\hline$C(19)-\operatorname{Si}(2)-C(31)$ & $106.56(7)$ \\
\hline $\mathrm{C}(25)-\mathrm{Si}(2)-\mathrm{S}(2)$ & $112.20(5)$ \\
\hline $\mathrm{C}(19)-\mathrm{Si}(2)-\mathrm{S}(2)$ & $111.55(5)$ \\
\hline $\mathrm{C}(31)-\mathrm{Si}(2)-\mathrm{S}(2)$ & $108.10(5)$ \\
\hline$C(6)-C(1)-C(2)$ & $117.32(15)$ \\
\hline$C(6)-C(1)-S i(1)$ & $122.53(13)$ \\
\hline$C(2)-C(1)-S i(1)$ & $120.14(12)$ \\
\hline$C(3)-C(2)-C(1)$ & $121.42(17)$ \\
\hline$C(4)-C(3)-C(2)$ & $120.31(19)$ \\
\hline$C(3)-C(4)-C(5)$ & $119.36(18)$ \\
\hline$C(4)-C(5)-C(6)$ & $120.5(2)$ \\
\hline$C(5)-C(6)-C(1)$ & $121.11(19)$ \\
\hline$C(8)-C(7)-C(12)$ & $117.75(15)$ \\
\hline $\mathrm{C}(8)-\mathrm{C}(7)-\mathrm{Si}(1)$ & $120.35(12)$ \\
\hline $\mathrm{C}(12)-\mathrm{C}(7)-\mathrm{Si}(1)$ & $121.86(12)$ \\
\hline $\mathrm{C}(9)-\mathrm{C}(8)-\mathrm{C}(7)$ & $120.86(18)$ \\
\hline $\mathrm{C}(10)-\mathrm{C}(9)-\mathrm{C}(8)$ & $120.39(19)$ \\
\hline$C(11)-C(10)-C(9)$ & $119.75(17)$ \\
\hline$C(10)-C(11)-C(12)$ & $120.35(18)$ \\
\hline $\mathrm{C}(11)-\mathrm{C}(12)-\mathrm{C}(7)$ & $120.86(17)$ \\
\hline$C(18)-C(13)-C(14)$ & $117.45(15)$ \\
\hline $\mathrm{C}(18)-\mathrm{C}(13)-\mathrm{Si}(1)$ & $122.96(12)$ \\
\hline C(14)-C(13)-Si(1) & $119.38(12)$ \\
\hline$C(15)-C(14)-C(13)$ & $121.23(18)$ \\
\hline$C(16)-C(15)-C(14)$ & $119.9(2)$ \\
\hline$C(17)-C(16)-C(15)$ & $120.11(17)$ \\
\hline$C(16)-C(17)-C(18)$ & $120.23(18)$ \\
\hline$C(13)-C(18)-C(17)$ & $121.02(17)$ \\
\hline$C(24)-C(19)-C(20)$ & $117.25(16)$ \\
\hline C(24)-C(19)-Si(2) & $122.49(13)$ \\
\hline C(20)-C(19)-Si(2) & $119.85(12)$ \\
\hline$C(21)-C(20)-C(19)$ & $121.59(18)$ \\
\hline$C(22)-C(21)-C(20)$ & $119.82(19)$ \\
\hline$C(21)-C(22)-C(23)$ & $120.29(19)$ \\
\hline
\end{tabular}




\begin{tabular}{|c|c|}
\hline$C(22)-C(23)-C(24)$ & $119.8(2)$ \\
\hline$C(23)-C(24)-C(19)$ & $121.21(19)$ \\
\hline$C(26)-C(25)-C(30)$ & $117.50(14)$ \\
\hline$C(26)-C(25)-S i(2)$ & $121.07(12)$ \\
\hline $\mathrm{C}(30)-\mathrm{C}(25)-\mathrm{Si}(2)$ & $121.21(12)$ \\
\hline$C(27)-C(26)-C(25)$ & $121.41(17)$ \\
\hline$C(28)-C(27)-C(26)$ & $120.08(17)$ \\
\hline$C(27)-C(28)-C(29)$ & $119.65(16)$ \\
\hline$C(28)-C(29)-C(30)$ & $120.51(17)$ \\
\hline$C(29)-C(30)-C(25)$ & $120.85(17)$ \\
\hline $\mathrm{C}(32)-\mathrm{C}(31)-\mathrm{C}(36)$ & $116.89(16)$ \\
\hline$C(32)-C(31)-S i(2)$ & $120.56(13)$ \\
\hline$C(36)-C(31)-S i(2)$ & $122.33(13)$ \\
\hline $\mathrm{C}(33)-\mathrm{C}(32)-\mathrm{C}(31)$ & $121.3(2)$ \\
\hline $\mathrm{C}(34)-\mathrm{C}(33)-\mathrm{C}(32)$ & $120.4(2)$ \\
\hline $\mathrm{C}(35)-\mathrm{C}(34)-\mathrm{C}(33)$ & $119.45(19)$ \\
\hline $\mathrm{C}(34)-\mathrm{C}(35)-\mathrm{C}(36)$ & $120.1(2)$ \\
\hline $\mathrm{C}(35)-\mathrm{C}(36)-\mathrm{C}(31)$ & $121.6(2)$ \\
\hline $\mathrm{N}(2)-\mathrm{C}(39)-\mathrm{C}(40)$ & $112.0(4)$ \\
\hline $\mathrm{N}(1)-\mathrm{C}(40)-\mathrm{C}(39)$ & $112.3(3)$ \\
\hline $\mathrm{C}(39 \mathrm{~A})-\mathrm{C}(40 \mathrm{~A})-\mathrm{N}(1)$ & $111(2)$ \\
\hline$C(40 A)-C(39 A)-N(2)$ & $122(3)$ \\
\hline $\mathrm{C}(2 \mathrm{~T})-\mathrm{C}(1 \mathrm{~T})-\mathrm{C}(6 \mathrm{~T})$ & 120.0 \\
\hline $\mathrm{C}(1 \mathrm{~T})-\mathrm{C}(2 \mathrm{~T})-\mathrm{C}(3 \mathrm{~T})$ & 120.0 \\
\hline $\mathrm{C}(2 \mathrm{~T})-\mathrm{C}(3 \mathrm{~T})-\mathrm{C}(4 \mathrm{~T})$ & 120.0 \\
\hline$C(5 \mathrm{~T})-\mathrm{C}(4 \mathrm{~T})-\mathrm{C}(3 \mathrm{~T})$ & 120.0 \\
\hline $\mathrm{C}(5 \mathrm{~T})-\mathrm{C}(4 \mathrm{~T})-\mathrm{C}(7 \mathrm{~T})$ & $123.9(8)$ \\
\hline $\mathrm{C}(3 \mathrm{~T})-\mathrm{C}(4 \mathrm{~T})-\mathrm{C}(7 \mathrm{~T})$ & $116.0(8)$ \\
\hline $\mathrm{C}(4 \mathrm{~T})-\mathrm{C}(5 \mathrm{~T})-\mathrm{C}(6 \mathrm{~T})$ & 120.0 \\
\hline$C(5 T)-C(6 T)-C(1 T)$ & 120.0 \\
\hline$C(2 S)-C(1 S)-C(6 S)$ & 120.0 \\
\hline$C(3 S)-C(2 S)-C(1 S)$ & 120.0 \\
\hline $\mathrm{C}(4 \mathrm{~S})-\mathrm{C}(3 \mathrm{~S})-\mathrm{C}(2 \mathrm{~S})$ & 120.0 \\
\hline$C(3 S)-C(4 S)-C(5 S)$ & 120.0 \\
\hline$C(6 S)-C(5 S)-C(4 S)$ & 120.0 \\
\hline$C(5 S)-C(6 S)-C(1 S)$ & 120.0 \\
\hline
\end{tabular}


Anisotropic displacement parameters $\left(\AA^{2} \times 10^{3}\right)$ for $\mathrm{Cd}(\mathrm{SSiPh} 3) 2(\mathrm{TMEDA})$. The anisotropic displacement factor exponent takes the form: $-2 \pi^{2}\left[h^{2} a^{* 2} U^{11}+\ldots+2 h k a^{*} b^{*} U^{12}\right]$

\begin{tabular}{|c|c|c|c|c|c|c|}
\hline & $\mathrm{U}^{11}$ & $\mathrm{U}^{22}$ & $\mathrm{U}^{33}$ & $\mathrm{U}^{23}$ & $\mathrm{U}^{13}$ & $\mathrm{U}^{12}$ \\
\hline $\mathrm{Cd}(1)$ & $22(1)$ & $22(1)$ & $29(1)$ & $-6(1)$ & $-10(1)$ & $2(1)$ \\
\hline $\mathrm{N}(1)$ & $31(1)$ & $33(1)$ & $29(1)$ & $-9(1)$ & $-12(1)$ & $3(1)$ \\
\hline $\mathrm{N}(2)$ & $23(1)$ & $38(1)$ & $41(1)$ & $-12(1)$ & $-13(1)$ & $4(1)$ \\
\hline $\mathrm{S}(1)$ & $33(1)$ & $23(1)$ & $26(1)$ & $-7(1)$ & $-7(1)$ & $-1(1)$ \\
\hline $\mathrm{S}(2)$ & $24(1)$ & $23(1)$ & $41(1)$ & $-3(1)$ & $-14(1)$ & $1(1)$ \\
\hline $\mathrm{Si}(1)$ & $26(1)$ & $21(1)$ & $26(1)$ & $-6(1)$ & $-10(1)$ & $3(1)$ \\
\hline $\operatorname{Si}(2)$ & $22(1)$ & $21(1)$ & $27(1)$ & $-4(1)$ & $-8(1)$ & $3(1)$ \\
\hline$C(1)$ & $30(1)$ & $26(1)$ & $34(1)$ & $-11(1)$ & $-14(1)$ & $7(1)$ \\
\hline$C(2)$ & $33(1)$ & $35(1)$ & $43(1)$ & $-7(1)$ & $-14(1)$ & $0(1)$ \\
\hline$C(3)$ & $31(1)$ & $47(1)$ & $63(1)$ & $-17(1)$ & $-17(1)$ & 2(1) \\
\hline$C(4)$ & $39(1)$ & $59(1)$ & $74(2)$ & $-24(1)$ & $-34(1)$ & $16(1)$ \\
\hline$C(5)$ & $50(1)$ & $71(2)$ & $52(1)$ & $-7(1)$ & $-32(1)$ & $15(1)$ \\
\hline$C(6)$ & $37(1)$ & $52(1)$ & $38(1)$ & $-5(1)$ & $-18(1)$ & $7(1)$ \\
\hline$C(7)$ & $30(1)$ & $26(1)$ & $24(1)$ & $-6(1)$ & $-9(1)$ & 1(1) \\
\hline$C(8)$ & $45(1)$ & $27(1)$ & $32(1)$ & $-6(1)$ & $-11(1)$ & 2(1) \\
\hline$C(9)$ & $62(1)$ & $30(1)$ & $41(1)$ & $1(1)$ & $-19(1)$ & $-9(1)$ \\
\hline$C(10)$ & $46(1)$ & $55(1)$ & $36(1)$ & $6(1)$ & $-15(1)$ & $-20(1)$ \\
\hline $\mathrm{C}(11)$ & $29(1)$ & $65(1)$ & $33(1)$ & $-3(1)$ & $-8(1)$ & $-4(1)$ \\
\hline $\mathrm{C}(12)$ & $30(1)$ & $41(1)$ & $31(1)$ & $-8(1)$ & $-10(1)$ & $4(1)$ \\
\hline $\mathrm{C}(13)$ & $35(1)$ & $24(1)$ & $27(1)$ & $-8(1)$ & $-13(1)$ & $7(1)$ \\
\hline$C(14)$ & $63(1)$ & $44(1)$ & $57(1)$ & $-28(1)$ & $-40(1)$ & $27(1)$ \\
\hline$C(15)$ & $85(2)$ & $61(1)$ & $71(2)$ & $-37(1)$ & $-55(1)$ & $50(1)$ \\
\hline$C(16)$ & $91(2)$ & $40(1)$ & $46(1)$ & $-22(1)$ & $-32(1)$ & $37(1)$ \\
\hline $\mathrm{C}(17)$ & $75(1)$ & $33(1)$ & $50(1)$ & $-23(1)$ & $-28(1)$ & $13(1)$ \\
\hline $\mathrm{C}(18)$ & $50(1)$ & $31(1)$ & $45(1)$ & $-16(1)$ & $-25(1)$ & $9(1)$ \\
\hline $\mathrm{C}(19)$ & $28(1)$ & $25(1)$ & $30(1)$ & $-7(1)$ & $-9(1)$ & $7(1)$ \\
\hline$C(20)$ & $40(1)$ & $28(1)$ & $33(1)$ & $-6(1)$ & $-7(1)$ & $0(1)$ \\
\hline $\mathrm{C}(21)$ & $63(1)$ & $43(1)$ & $30(1)$ & $-9(1)$ & $-7(1)$ & $7(1)$ \\
\hline$C(22)$ & $74(2)$ & $66(1)$ & $38(1)$ & $-26(1)$ & $-26(1)$ & $31(1)$ \\
\hline
\end{tabular}




\begin{tabular}{|c|c|c|c|c|c|c|}
\hline$C(23)$ & $49(1)$ & $99(2)$ & $59(1)$ & $-42(1)$ & $-32(1)$ & $21(1)$ \\
\hline$C(24)$ & $31(1)$ & $72(1)$ & $42(1)$ & $-24(1)$ & $-13(1)$ & $6(1)$ \\
\hline$C(25)$ & $21(1)$ & $27(1)$ & $30(1)$ & $-3(1)$ & $-10(1)$ & $2(1)$ \\
\hline$C(26)$ & $25(1)$ & $27(1)$ & $41(1)$ & $-5(1)$ & $-8(1)$ & $3(1)$ \\
\hline$C(27)$ & $31(1)$ & $28(1)$ & $56(1)$ & $1(1)$ & $-15(1)$ & $0(1)$ \\
\hline$C(28)$ & $28(1)$ & $41(1)$ & $43(1)$ & $11(1)$ & $-14(1)$ & $-6(1)$ \\
\hline$C(29)$ & $33(1)$ & $56(1)$ & $30(1)$ & $-3(1)$ & $-7(1)$ & $-4(1)$ \\
\hline$C(30)$ & $30(1)$ & $39(1)$ & $32(1)$ & $-8(1)$ & $-9(1)$ & $-1(1)$ \\
\hline$C(31)$ & $31(1)$ & $24(1)$ & $30(1)$ & $-5(1)$ & $-8(1)$ & $5(1)$ \\
\hline$C(32)$ & $33(1)$ & $29(1)$ & $87(2)$ & $-18(1)$ & $-23(1)$ & $9(1)$ \\
\hline$C(33)$ & $40(1)$ & $34(1)$ & $113(2)$ & $-21(1)$ & $-23(1)$ & $16(1)$ \\
\hline$C(34)$ & $55(1)$ & $32(1)$ & $77(2)$ & $-23(1)$ & $-6(1)$ & $13(1)$ \\
\hline$C(35)$ & $76(2)$ & $50(1)$ & $81(2)$ & $-42(1)$ & $-34(1)$ & $17(1)$ \\
\hline$C(36)$ & $61(1)$ & $50(1)$ & $68(1)$ & $-35(1)$ & $-37(1)$ & 21(1) \\
\hline$C(37)$ & $27(1)$ & $49(1)$ & $77(1)$ & $-27(1)$ & $-20(1)$ & $12(1)$ \\
\hline$C(38)$ & $27(1)$ & $43(1)$ & $84(2)$ & $-22(1)$ & $-14(1)$ & $-3(1)$ \\
\hline$C(39)$ & $30(1)$ & $79(2)$ & $45(1)$ & $-13(2)$ & $-21(1)$ & $18(1)$ \\
\hline$C(40)$ & $38(1)$ & $64(2)$ & $41(1)$ & $-21(1)$ & $-19(1)$ & $-1(1)$ \\
\hline $\mathrm{C}(40 \mathrm{~A})$ & $36(7)$ & $63(15)$ & $46(8)$ & $-11(8)$ & $-27(6)$ & $12(7)$ \\
\hline$C(39 A)$ & $33(9)$ & $110(20)$ & $73(13)$ & $-30(15)$ & $-44(9)$ & $27(12)$ \\
\hline $\mathrm{C}(41)$ & $102(2)$ & $41(1)$ & $29(1)$ & $-3(1)$ & $-11(1)$ & $-14(1)$ \\
\hline$C(42)$ & $71(2)$ & $59(1)$ & $42(1)$ & $-26(1)$ & $-17(1)$ & $25(1)$ \\
\hline $\mathrm{C}(1 \mathrm{~T})$ & $56(6)$ & $37(5)$ & $46(6)$ & $-10(5)$ & $-11(5)$ & $-4(4)$ \\
\hline $\mathrm{C}(2 \mathrm{~T})$ & $51(5)$ & $38(5)$ & $64(6)$ & $-11(4)$ & $-18(5)$ & $2(5)$ \\
\hline $\mathrm{C}(3 \mathrm{~T})$ & $43(6)$ & $39(5)$ & $39(5)$ & $-14(4)$ & $-8(4)$ & $1(5)$ \\
\hline $\mathrm{C}(4 \mathrm{~T})$ & $45(5)$ & $32(5)$ & $39(5)$ & $-17(4)$ & $-7(3)$ & $2(4)$ \\
\hline $\mathrm{C}(5 \mathrm{~T})$ & $63(8)$ & $41(6)$ & $37(6)$ & $-19(5)$ & $-15(5)$ & $2(6)$ \\
\hline $\mathrm{C}(6 \mathrm{~T})$ & $73(8)$ & $38(5)$ & $45(5)$ & $-15(4)$ & $-28(6)$ & $2(5)$ \\
\hline $\mathrm{C}(7 \mathrm{~T})$ & $39(4)$ & $49(5)$ & $50(5)$ & $-15(4)$ & $-28(4)$ & $27(4)$ \\
\hline$C(1 S)$ & $48(7)$ & $56(11)$ & $54(6)$ & $-25(7)$ & $-18(6)$ & $10(8)$ \\
\hline$C(2 S)$ & $43(5)$ & $74(8)$ & $40(5)$ & $-10(5)$ & $-14(4)$ & $-15(5)$ \\
\hline$C(3 S)$ & $30(4)$ & $42(5)$ & $64(6)$ & $-25(5)$ & $-10(5)$ & $0(4)$ \\
\hline$C(4 S)$ & $99(8)$ & $41(7)$ & $46(6)$ & $-19(5)$ & $26(5)$ & $-24(5)$ \\
\hline$C(5 S)$ & $122(12)$ & $46(12)$ & $40(6)$ & $-6(9)$ & $-35(7)$ & $-19(11)$ \\
\hline$C(6 S)$ & 102(8) & $38(6)$ & $83(8)$ & $-27(6)$ & $-51(7)$ & $5(7)$ \\
\hline
\end{tabular}


Hydrogen coordinates ( x 104) and isotropic displacement parameters $\left(\AA^{2} \times 10^{3}\right)$ for $\mathrm{Cd}$ (SSiPh3)2(TMEDA).

\begin{tabular}{|c|c|c|c|c|}
\hline & $\mathrm{x}$ & $\mathrm{y}$ & z & $\mathrm{U}(\mathrm{eq})$ \\
\hline $\mathrm{H}(2 \mathrm{~A})$ & 3613 & 5608 & 2144 & 45 \\
\hline $\mathrm{H}(3 \mathrm{~A})$ & 5602 & 5561 & 2495 & 56 \\
\hline $\mathrm{H}(4 \mathrm{~A})$ & 5661 & 4585 & 3935 & 63 \\
\hline $\mathrm{H}(5 \mathrm{~A})$ & 3719 & 3642 & 5019 & 68 \\
\hline $\mathrm{H}(6 \mathrm{~A})$ & 1720 & 3684 & 4676 & 51 \\
\hline $\mathrm{H}(8 \mathrm{~A})$ & 622 & 2543 & 3818 & 43 \\
\hline $\mathrm{H}(9 \mathrm{~A})$ & -1061 & 1322 & 4855 & 56 \\
\hline $\mathrm{H}(10 \mathrm{~A})$ & -3139 & 1750 & 5672 & 60 \\
\hline $\mathrm{H}(11 \mathrm{~A})$ & -3544 & 3402 & 5439 & 54 \\
\hline $\mathrm{H}(12 \mathrm{~A})$ & -1891 & 4638 & 4371 & 41 \\
\hline $\mathrm{H}(14 \mathrm{~A})$ & -1197 & 5745 & 2411 & 56 \\
\hline $\mathrm{H}(15 \mathrm{~A})$ & -2215 & 7198 & 2474 & 74 \\
\hline $\mathrm{H}(16 \mathrm{~A})$ & -1426 & 8293 & 3081 & 66 \\
\hline $\mathrm{H}(17 \mathrm{~A})$ & 282 & 7892 & 3708 & 59 \\
\hline $\mathrm{H}(18 \mathrm{~A})$ & 1295 & 6425 & 3672 & 46 \\
\hline $\mathrm{H}(20 \mathrm{~A})$ & 1208 & 515 & 4460 & 43 \\
\hline $\mathrm{H}(21 \mathrm{~A})$ & 1273 & 333 & 5948 & 57 \\
\hline $\mathrm{H}(22 \mathrm{~A})$ & 3246 & 774 & 6147 & 66 \\
\hline $\mathrm{H}(23 \mathrm{~A})$ & 5139 & 1473 & 4856 & 74 \\
\hline $\mathrm{H}(24 \mathrm{~A})$ & 5069 & 1688 & 3359 & 56 \\
\hline $\mathrm{H}(26 \mathrm{~A})$ & 3810 & 3150 & 2312 & 39 \\
\hline $\mathrm{H}(27 \mathrm{~A})$ & 5083 & 4480 & 1095 & 49 \\
\hline $\mathrm{H}(28 \mathrm{~A})$ & 6349 & 4252 & -324 & 50 \\
\hline $\mathrm{H}(29 \mathrm{~A})$ & 6358 & 2683 & -514 & 51 \\
\hline $\mathrm{H}(30 \mathrm{~A})$ & 5067 & 1347 & 691 & 41 \\
\hline $\mathrm{H}(32 \mathrm{~A})$ & 5444 & -10 & 2794 & 58 \\
\hline $\mathrm{H}(33 \mathrm{~A})$ & 6254 & -1509 & 2654 & 76 \\
\hline $\mathrm{H}(34 \mathrm{~A})$ & 5135 & -2543 & 2168 & 69 \\
\hline $\mathrm{H}(35 \mathrm{~A})$ & 3078 & -2154 & 1980 & 74 \\
\hline $\mathrm{H}(36 \mathrm{~A})$ & 2268 & -649 & 2101 & 62 \\
\hline
\end{tabular}




\begin{tabular}{|c|c|c|c|c|}
\hline $\mathrm{H}(37 \mathrm{~A})$ & -3121 & 3219 & 2335 & 73 \\
\hline $\mathrm{H}(37 \mathrm{~B})$ & -1854 & 3989 & 2062 & 73 \\
\hline $\mathrm{H}(37 \mathrm{C})$ & -2231 & 3092 & 3003 & 73 \\
\hline $\mathrm{H}(38 \mathrm{~A})$ & -2867 & 1632 & 2231 & 77 \\
\hline $\mathrm{H}(38 \mathrm{~B})$ & -1961 & 1404 & 2893 & 77 \\
\hline $\mathrm{H}(38 \mathrm{C})$ & -1415 & 1244 & 1884 & 77 \\
\hline $\mathrm{H}(39 \mathrm{~A})$ & -1028 & 3816 & 664 & 61 \\
\hline $\mathrm{H}(39 \mathrm{~B})$ & -2055 & 2937 & 762 & 61 \\
\hline $\mathrm{H}(40 \mathrm{~A})$ & -205 & 1944 & 436 & 53 \\
\hline $\mathrm{H}(40 \mathrm{~B})$ & -68 & 2908 & -419 & 53 \\
\hline $\mathrm{H}(40 \mathrm{C})$ & -143 & 3939 & 166 & 55 \\
\hline $\mathrm{H}(40 \mathrm{D})$ & -78 & 3202 & -444 & 55 \\
\hline $\mathrm{H}(39 \mathrm{C})$ & -837 & 1982 & 834 & 79 \\
\hline $\mathrm{H}(39 \mathrm{D})$ & -1849 & 2800 & 682 & 79 \\
\hline $\mathrm{H}(41 \mathrm{~A})$ & 2697 & 4108 & -296 & 92 \\
\hline $\mathrm{H}(41 \mathrm{~B})$ & 1200 & 4434 & -135 & 92 \\
\hline $\mathrm{H}(41 \mathrm{C})$ & 1920 & 4014 & -989 & 92 \\
\hline $\mathrm{H}(42 \mathrm{~A})$ & 2322 & 2359 & -745 & 83 \\
\hline $\mathrm{H}(42 \mathrm{~B})$ & 1817 & 1592 & 278 & 83 \\
\hline $\mathrm{H}(42 \mathrm{C})$ & 3071 & 2383 & -19 & 83 \\
\hline $\mathrm{H}(1 \mathrm{TA})$ & 12582 & -473 & 9685 & 58 \\
\hline $\mathrm{H}(2 \mathrm{TA})$ & 11941 & 384 & 8413 & 63 \\
\hline $\mathrm{H}(3 \mathrm{TA})$ & 9707 & 745 & 8635 & 49 \\
\hline $\mathrm{H}(5 \mathrm{TA})$ & 8753 & -608 & 11401 & 55 \\
\hline $\mathrm{H}(6 \mathrm{TA})$ & 10988 & -968 & 11179 & 59 \\
\hline $\mathrm{H}(7 \mathrm{TA})$ & 7628 & 764 & 9497 & 65 \\
\hline $\mathrm{H}(7 \mathrm{~TB})$ & 7446 & 862 & 10508 & 65 \\
\hline $\mathrm{H}(7 \mathrm{TC})$ & 7005 & -161 & 10400 & 65 \\
\hline $\mathrm{H}(1 \mathrm{SA})$ & 11422 & 373 & 8617 & 61 \\
\hline $\mathrm{H}(2 \mathrm{SA})$ & 9447 & 1002 & 8407 & 65 \\
\hline $\mathrm{H}(3 \mathrm{SA})$ & 7503 & 820 & 9695 & 53 \\
\hline $\mathrm{H}(4 \mathrm{SA})$ & 7534 & 8 & 11192 & 88 \\
\hline $\mathrm{H}(5 \mathrm{SA})$ & 9509 & -622 & 11401 & 82 \\
\hline $\mathrm{H}(6 \mathrm{SA})$ & 11453 & -439 & 10114 & 80 \\
\hline
\end{tabular}

